\title{
Novel DNA-Based Molecules and Their Charge Transport Properties
}

\author{
Danny Porath ${ }^{1 *}$ \\ 1) Institute of Chemistry and Center for Nanoscience and Nanotechnology, The Hebrew University of Jerusalem, \\ 91904 Israel \\ ${ }^{*}$ E-mail danny.porath@mail.huji.ac.il
}

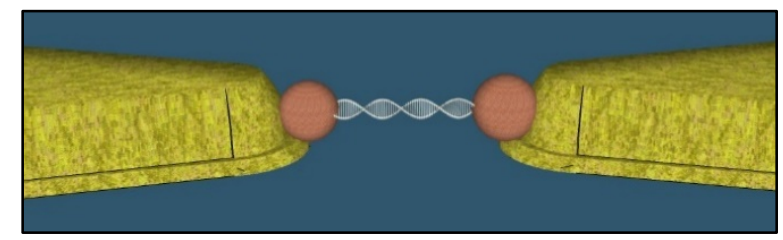

Charge transport through molecular structures is interesting both scientifically and technologically. To date, DNA is the only type of polymer that transports significant currents over distances of more than a few nanometers in individual molecules. Nevertheless and in spite of large efforts to elucidate the charge transport mechanism through DNA a satisfying characterization and mechanistic description has not been provided yet. For molecular electronics, DNA derivatives are by far more promising than native DNA due to their improved charge-transport properties.

In recent years we have invested great efforts to address the above issues. Measuring the charge transport in DNA was elusive due to great technical difficulties leading to carious results. We were recently able to devise an experiment in which double-stranded DNA is well positioned between metal electrodes. Electrical measurements give surprisingly high currents over 100 base-pairs $(\sim 30 \mathrm{~nm})$ elevated from the surface. The temperature dependence indicates backbone-related band-like transport.

In collaboration with the Kotlyar group, We were also able to synthesize and measure long (hundreds of nanometers) DNA-based derivatives that transport significant currents when deposited on hard substrates. Among the molecules metal containing DNA which is true metalorganic hybrid, a smooth and thin metal coated DNA and G-quadruplex DNA.

Step by step we improve the synthesized constructs and the measurement methods of single DNA-based molecules. I will present new and surprising results on dsDNA molecules. I will present new DNA-based molecules and report on our measurements of their properties.

\section{References.}

1. D. Porath, A. Bezryadin, S. de Vries and C. Dekker, Nature 403, 635 (2000).

2. Danny Porath, Rosa Di Felice and Gianaurelio Cuniberti, Topics in Current Chemistry Vol. 237, pp. 183-228 Ed. Gary Shuster. Springer Verlag, 2004.

3. Alexander Kotlyar, Nataly Borovok, Tatiana Molotsky, Hezy Cohen, Errez Shapir and Danny Porath, Advanced Materials 17, 1901 (2005).

4. Errez Shapir et al., Nature Materials 7, 68 (2008).

5. Gideon I. Livshits, Jamal Ghabboun, Natalia Borovok, Alexander B. Kotlyar, Danny Porath, Advanced materials 26, 4981 (2014).

6. GI. Livshits et al., Nature Nanotechnology 9, 1040 (2014).

7. G. Eidelshtein et al., Advanced Materials 28, 4839 (2016).

8. Avigail Stern, Gennady Eidelstein, Roman Zhuravel, Gideon I. Livshits, Dvir. Rotem, Alexander B. Kotlyar and Danny Porath, Advanced Materials 30, 1800433 (2018).

9. R. Zhuravel, A. Stern, N. Fardian-Melamed, G. Eidelshtein, L. Katrivas, D. Rotem, A. Kotlyar and D. Porath, Advanced Materials Online (2018). 